\title{
SQUID-Based Magnetic Resonance Imaging at Ultra-Low Field Using the Backprojection Method
}

\author{
Qingqian Guo, ${ }^{1,2}$ Changyu Ma, ${ }^{2}$ Xin Zhang, ${ }^{1,2}$ Yajie Xu $\mathbb{D}^{\circ},{ }^{2}$ Meisheng Fan, ${ }^{2}$ Peng Yu, ${ }^{2}$ \\ Tao Hu, ${ }^{2}$ Yan Chang $\mathbb{D}^{2},{ }^{2}$ and Xiaodong Yang $\mathbb{D}^{2}$ \\ ${ }^{1}$ School of Biomedical Engineering (Suzhou), Division of Life Sciences and Medicine, \\ University of Science and Technology of China, Hefei, Anhui 230026, China \\ ${ }^{2}$ Suzhou Institute of Biomedical Engineering and Technology, Chinese Academy of Science, Suzhou, Jiangsu 215163, China
}

Correspondence should be addressed to Yan Chang; changy@sibet.ac.cn and Xiaodong Yang; xiaodong.yang@sibet.ac.cn

Received 19 May 2020; Revised 26 August 2020; Accepted 18 September 2020; Published 22 October 2020

Academic Editor: Lars G. Hanson

Copyright (C) 2020 Qingqian Guo et al. This is an open access article distributed under the Creative Commons Attribution License, which permits unrestricted use, distribution, and reproduction in any medium, provided the original work is properly cited.

Ultra-low field magnetic resonance imaging (ULF MRI) is an effective imaging technique that applies the ultrasensitive detector of superconducting quantum interference device (SQUID) sensor to detect the MR signal at a microtesla field range. In this work, we designed and developed a SQUID-based ULF MRI system with a frequency-adjustable measurement field, the performance of which was characterized via water phantoms. In order to enhance the MR signals, a $500 \mathrm{mT}$ Halbach magnet was used to prepolarize the magnetization of the sample prior to excitation. The signal-to-noise-ratio (SNR) of the spin-echo- (SE-) based pulse sequence can reach up to 70 in a single scan. The images were then reconstructed successfully by using the maximum likelihood expectation maximization (MLEM) algorithm based on the backprojection imaging method. It was demonstrated that an in-plane resolution of $1.8 \times 1.8 \mathrm{~mm}^{2}$ can be achieved which indicated the feasibility of SQUID-based MRI at the ULF.

\section{Introduction}

Conventional magnetic resonance imaging (MRI) uses high magnetic fields, gradient fields, and radio frequency pulses to generate images of the organs in the body as one of the most important methods in clinical investigations. Besides, it has been widely used in physics, chemistry, biology, and medicine [1-4]. MRI signals are obtained by Faraday electromagnetic induction and the strength scales linearly with the square of the magnetic field strength [5]. Thus, traditional MRI commonly uses the superconducting magnet to improve the signal-to-noise-ratio (SNR) [6]. However, the homogeneity could be decreased with the increment of the strength of the magnetic field. The precession fields employed in MRI over the past ten years have risen from $1.5 \mathrm{~T}$ to the current $3 \mathrm{~T}$ clinical standard. Research systems even employ fields of up to $14 \mathrm{~T}$ [7-9]. There are, however, many MRI applications where the ultra-high field is not an optimal choice, for example, imaging performed in the presence of metal or where it is impractical to employ a large and expensive magnet [10]. In contrast, MRI at ultra-low fields (ULF MRI) has been under development for decades and exhibits various advantages, for instance, fewer artifacts generated by the inhomogeneity of the magnetic field, smaller size, and lower cost of the system $[1,11]$. ULF magnetic resonance technology offers a wide range of applications in chemistry, physiology, and biomedicine, such as imaging of water, the human brain, the human forearm, and the human wrist [12-14].

Although many conventional MRI experiments are conducted based on Faraday induction coils because of their low cost and simple preparation, the SNR will be decreased greatly in ULF MRI since the strength of the measurement field is much smaller $(1 \% \circ)$ compared with the conventional high-field MRI. In order to counteract the loss of SNR in ULF MRI, it is necessary to explore a new method for the acquisition of the weak magnetic resonance signal to improve the sensitivity $[11,15]$. To mitigate the sensitivity loss of Faraday induction, superconducting quantum interference devices (SQUIDs) are used to detect MR signals 
$[10,11]$. SQUIDs made from low-transition temperature superconductors are the most sensitive detectors of the magnetic field [15-17]. When coupled to an untuned superconducting magnetometer or gradiometer, they become extremely sensitive detectors of magnetic fields, achieving magnetic field noise in the order of $1 \mathrm{ft} / \sqrt{\mathrm{Hz}}$ $[13,15,18,19]$.

In recent years, research on SQUID-based ULF MRI has received widespread attention. Espy et al. used a 7-channel ULF MRI system for the detection of hazardous material and the human brain $[20,21]$. Clarke et al. presented highresolution images of phantoms and bell peppers in ULF of $132 \mu \mathrm{T}[18,22]$. Moreover, they also acquired images of the human brain based on the ULF MRI system operating in a magnetic field of $130 \mu \mathrm{T}[23,24]$. Espy et al. constructed images from many kinds of samples including the human brain, human hand, liquid explosives, and water by SQUID in microtesla magnetic fields, and the resolution was sufficient to reveal anatomical features $[25,26]$. Huang et al. acquired both 1-D and 2-D MR images with high quality by applying three orthogonal SQUIDs as reference channels to eliminate stripe-artifacts in ULF MRI by measurement of water phantom [27]. Vogel et al. have demonstrated an encoding magnet array moving around the sample for the generation of a 3-D image by backprojection [28].

In this paper, we designed and developed a SQUIDbased ULF MRI system, and imaging experiments were conducted to characterize the performance of the system by using multirods inside the phantom with different diameters. The SNR of the spin-echo (SE) signal could reach up to 70 in a single measurement. The system achieves an in-plane resolution of $1.8 \times 1.8 \mathrm{~mm}^{2}$ based on the backprojection imaging method.

\section{Materials and Methods}

2.1. ULF MRI System Design. The ULF MRI system is shown schematically in Figure 1(a). The entire system is enclosed in a room $\left(3.03 \times 2.8 \times 2.75 \mathrm{~m}^{3}\right)$ constructed of $8 \mathrm{~mm}$ thick aluminum sheets which can provide RF shielding in the range of $2 \sim 9 \mathrm{kHz}$ (above $20 \mathrm{~dB}$ ). The ultra-low measurement field, $B_{\mathrm{m}}$, was generated using a pair of Helmholtz coils with a diameter of $1.35 \mathrm{~m}[13,29,30]$. The strength of the $B_{\mathrm{m}}$ field is $100 \sim 200 \mu \mathrm{T}$, which produces a resonance frequency of $4.25 \sim 8.5 \mathrm{kHz}$ for proton spins. Two sets of gradient coils, $G_{x}$ $\left(\mathrm{d} B_{z} / \mathrm{d} x\right)$ and $G_{y}\left(\mathrm{~d} B_{z} / \mathrm{d} y\right)$, are used to provide gradient fields in the two transverse directions, with a size of $1.45 \times 1.02 \mathrm{~m}^{2}$ and $0.9 \times 1.28 \mathrm{~m}^{2}$, respectively. The gradient field $G_{z}\left(\mathrm{~d} B_{z} / \mathrm{d} z\right)$ is generated by a set of Maxwell coils with a diameter of $1.2 \mathrm{~m}$. All the gradient fields range from $100 \sim 400 \mu \mathrm{T} / \mathrm{m}$. A three-layer Halbach magnet is used to prepolarize the samples (Figure 1(b)) with a field strength of $500 \mathrm{mT}\left(B_{p}\right)$. The Halbach magnet is a novel type of permanent magnet constructed by magnetic blocks that are magnetized in different directions and arranged to provide a uniform field that is largely contained inside the array [31]. During the prepolarization, the phantom is placed at the central area $(\sim 500 \mathrm{mT})$ of the cylindrical hole inside the Halbach magnet (Figure 2(b)). In order to quickly move the sample from the magnet to the SQUID sensor after prepolarization, a slide rail made of polymethyl methacrylate is placed between the Halbach magnet and the measuring position below the SQUID. The magnet and slide rail are supported by two wooden boxes with the same height $(0.83 \mathrm{~m})$ in front and back (Figure 2(a)), respectively. Before each measurement, the sample is prepolarized for $15 \mathrm{~s}$ to enhance the $\mathrm{MR}$ signals. According to the $T_{1}$ (about $4 \mathrm{~s}$ ) of water, the polarization efficiency can reach more than 97\%. After the prepolarization, the pulse sequence is triggered by a photoelectric switch as soon as the phantom shifts to the detection area. The distance between magnet and SQUID is $1.10 \mathrm{~m}$, and the transport time of the sample is less than $800 \mathrm{~ms}$, corresponding to a nominal prepolarized field of $290 \mathrm{mT}$ because the decay of magnetization during the transportation.

Figure 3 depicts the data acquisition process performed by the ULF MRI system, where the gradient field always exists during the experiment and the $B_{1}$ pulse is applied to generate the magnetic resonance signal. In this work, the Supracon's CE2S blue type SQUID sensor is selected for the measurement of magnetic resonance signals produced by the pulse sequence [23]. The CE2S blue type SQUID with parameters of $L_{\mathrm{in}}=420 \mathrm{nH}$ inputs coil inductance and $0.26 \mu \mathrm{A} /$ $\Phi_{0}\left(\Phi_{0}=h / 2 e \approx 2.07 \times 10^{-15} \mathrm{Tm}^{2}\right)$ inputs current sensitivity. The second-order gradiometer wound as 1-2-1 turns, with $50 \mathrm{~mm}$ baseline and $50 \mathrm{~mm}$ loop diameters, was connected to the input coil integrated onto the SQUID chip. The flux noise is $10.7 \mu \Phi_{0} / \sqrt{\mathrm{Hz}}$ in addition to the SQUID's intrinsic $3 \mu \Phi_{0} / \sqrt{\mathrm{Hz}}$ inside the SQUID loop. The SQUID is based on $\mathrm{Nb}$ thin films and enclosed in a superconducting $\mathrm{Nb}$ shield to protect them from external magnetic noise. The magnetic field noise of the detector referred to the lowest loop is $0.8 \mathrm{ft} / \sqrt{\mathrm{Hz}}$, and the distance between this lowest loop and the room temperature outside surface is less than $20 \mathrm{~mm}$.

2.2. Backprojection Imaging. Backprojection is an extension of the frequency encoding procedure. The goal of backprojection imaging is to obtain a $2-\mathrm{D}$ representation of the internal structure of an object by gradient fields from different angles. The gradient fields are constant and applied at different angles of the desired slice. Only projections from 0 to 180 degrees are considered because the projections differing by 180 degrees are mirror images of each other (Figure 4). The gradient angle is determined by a linear combination of two gradient fields in different directions. This approach is to backproject each projection and sum all the backprojections to generate a slice [28, 32].

As can be seen from Figure 4, to obtain a YZ plane image of the sample, the frequency encoding gradient $\left(G_{f}\right)$ can be decomposed into orthogonal gradient directions $\left(G_{z}\right.$ and $\left.G_{y}\right)$ in the following proportions:

$$
\begin{aligned}
& G_{z}=G_{f} \sin \theta, \\
& G_{y}=G_{f} \cos \theta .
\end{aligned}
$$

A linear combination of the two orthogonal gradients is applied, but only the combined gradient direction changes, 


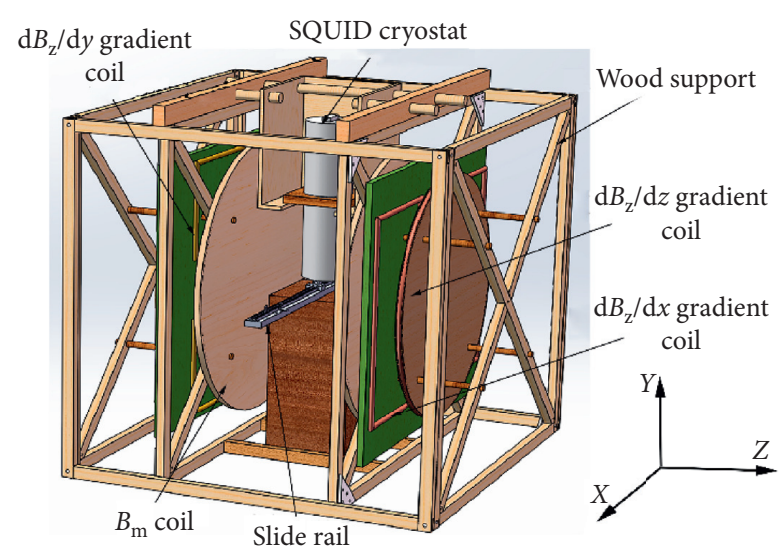

(a)

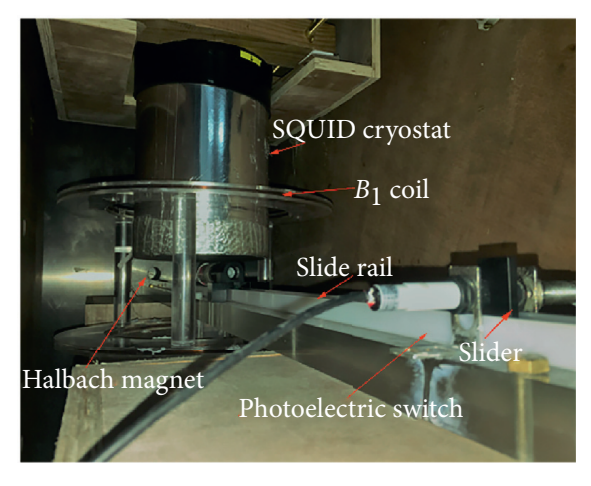

(b)

Figure 1: Measurement configuration of the ULF MRI system. (a) Schematic diagram of magnetic field coils and gradient coils. (b) Photograph of the transporting system.

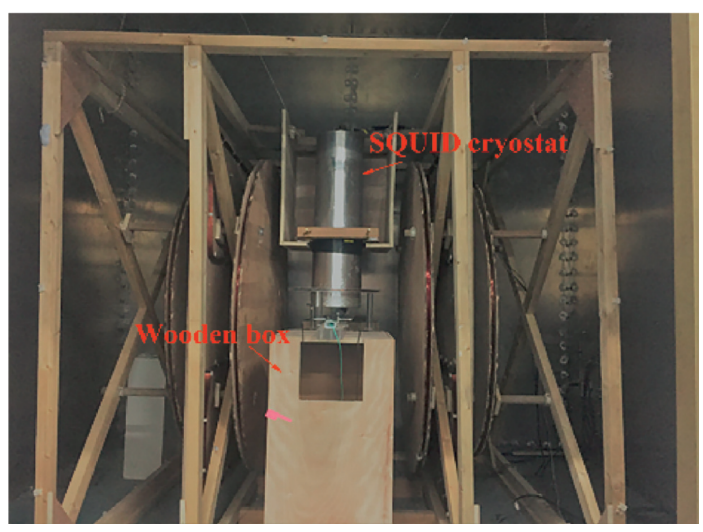

(a)

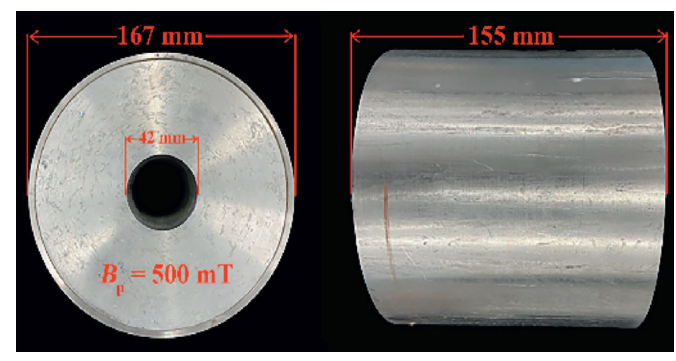

(b)

Figure 2: (a) Photograph of the ULF MRI system. (b) A three-layer Halbach magnet with a field strength of $500 \mathrm{mT}$.

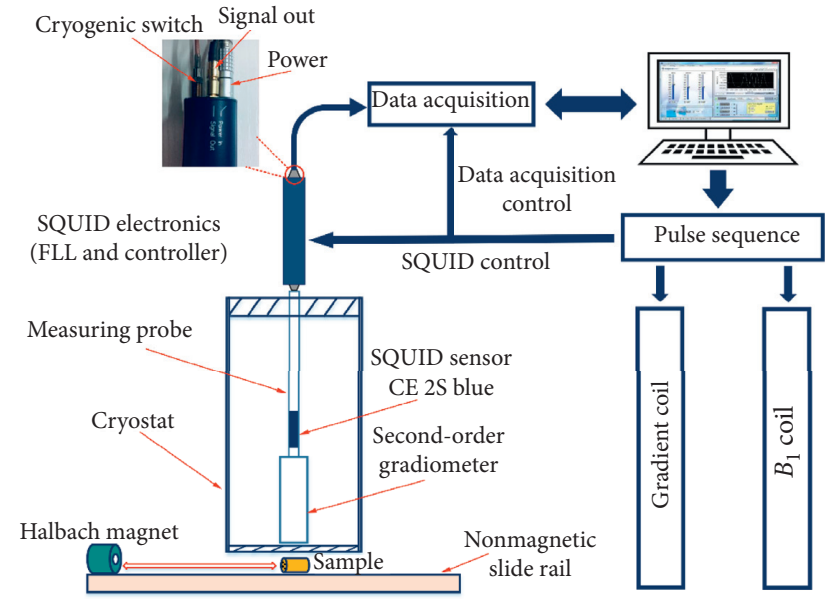

FIgURE 3: Schematic diagram of the data acquisition system.

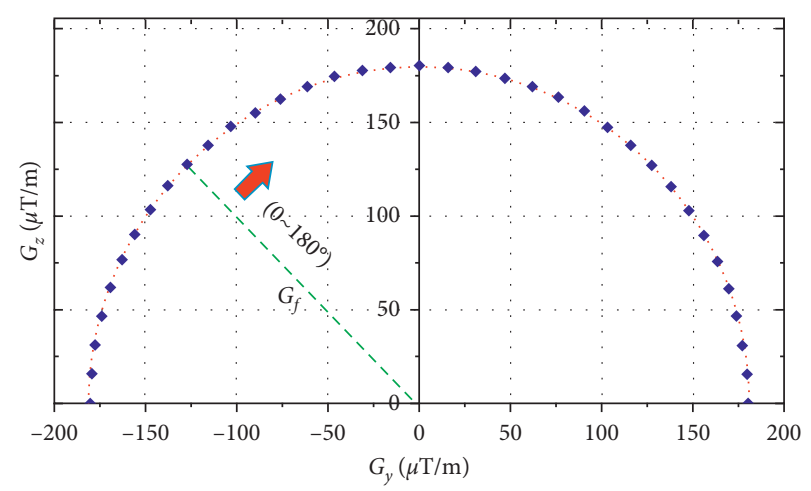

FIgURE 4: Schematic illustration of the backprojection imaging method. 
e.g., the angle increment is about 4.98 degrees for 36 projections.

2.3. Pulse Sequence. To increase the effective acquisition time, a spin echo- (SE-) based pulse sequence was designed as shown in Figure 5. The phantom was first prepolarized in the Halbach magnet for $15 \mathrm{~s}$, and then it was quickly transported to the measuring position. The prepolarizing field $\left(B_{p}\right)$ experienced by the sample is reduced adiabatically as the sample moves out of the Halbach array and over to the measurement position [24]. Once the sample arrived, the photoelectric switch was immediately triggered and a TTL signal was generated to start the excitation and the following signal acquisition. Note that two constant gradient fields $\left(G_{y}\right.$ and $G_{z}$ ) were applied throughout the whole scan. A $\pi / 2$ pulse generated by a pair of coils was applied orthogonally to the measurement field to rotate the proton spins to start their precession. Subsequently, a $\pi$ pulse is applied to obtain an echo signal. The time of echo (TE) is variable during the experiment. The gradient direction is determined by varying $G_{y}$ and $G_{z}$ through successive values. After the signal was recorded, the sample was transported back to the magnet for the next measurement.

Note that the SQUID sensor provides a voltage output that is linear with the detected magnetic field in a small range. Since the amplitude of the oscillating magnetic field generated by the $B_{1}$ pulse is much larger than the signal, this will lead to a shift of the working point of the SQUID sensor. Consequently, the SQUID CTRL (cryogenic switch control of the SQUID) was applied to ensure the stability of the working point. During the $B_{1}$ excitation, the cryogenic switch will be activated to bring the input circuit of SQUID above its superconducting transition temperature, which will disconnect the circuit coupled to SQUID.

\section{Results}

3.1. SNR Evaluation. SNR is the primary limiting factor for MRI. To evaluate the SNR of the designed system, a free induction decay (FID) signal was obtained in a single scan using the FID-based pulse sequence (Figure 6). Similar to the pulse sequence discussed above (Figure 5), a $\pi / 2$ pulse was applied for $2 \mathrm{~ms}$ after prepolarization. The cryogenic switch was turned on during the $\pi / 2$ pulse to avoid the large field shock to the SQUID sensor, and subsequently the signal was acquired. As illustrated in Figure 7, the signal duration is about $500 \mathrm{~ms}$ (Figure 7(a)) and the SNR is about 11.5 at $5.5 \mathrm{kHz}$ (Figure 7(b)). The decay of the FID signal is exponential. The fitting curve is plotted in Figure 8. The equation of $A_{\mathrm{FID}}=21.66(\mathrm{pT}) \cdot e^{-0.0059 t}\left(A_{\mathrm{FID}}\right.$ is the amplitude of the FID signal, $t$ is the acquisition time) was obtained with the square of the correlation coefficient, $R^{2}$, being 0.99 .

The measurement field of the ULF MRI system was provided by a pair of Helmholtz coils at the room temperature, which allows the measurement field to be adjusted. Figure 9 depicts the relationship between the measured Larmor frequency, $f_{\mathrm{L}}$, and the current, $I$. The linear equation of $f_{\mathrm{L}}=5.0506(\mathrm{kHz} / \mathrm{A}) \cdot I(\mathrm{~A})+0.8357(\mathrm{kHz})$ was obtained with the square of the correlation coefficient, $R^{2}$, of 0.9996 . The fitted straight line verified the ULF MRI system with a frequency-adjustable measurement field and provided a precise relationship of the measurement field. It is also obvious that the vertical intercept $(0.8357 \mathrm{kHz})$ is nonzero, which indicates that the earth field in the horizontal plane (XZ) with an equivalent of $19.6 \mu \mathrm{T}$ contributes to the static magnetic field.

Further, we used an SE-based pulse sequence (Figure 6) to evaluate the SNR of a single scan since the spin echo will eliminate the decay due to the local field inhomogeneity. The measured signal was plotted in Figure 10 without averaging, which depicts that the SE signal duration is about $900 \mathrm{~ms}$ (Figure 10(a)) and the SNR is about $\sim 70$ at $5.46 \mathrm{kHz}$ (Figure 10(b)). Consequently, the SE-based sequence was selected to conduct the experiment for the significantly improved signal duration and SNR.

3.2. Phantom Experiment. Three phantoms were used to characterize the performance of the ULF MRI system by applying an SE-based pulse sequence (Figure 5). Figure 11(a) is a photograph of a $35 \mathrm{~mm}$ diameter by $52 \mathrm{~mm}$ height cylindrical plastic phantom which consists of seven rods with a diameter of $5 \mathrm{~mm}$. The phantom was filled with pure water with a resistivity of $18.2 \mathrm{M} \Omega / \mathrm{cm}$ at $25^{\circ} \mathrm{C}$. By calculating the frequency resolution and image resolution for the phantom (Table 1), it can be concluded that the longer the acquisition time $(51.2 \mathrm{~ms}, 102.4 \mathrm{~ms}$, and $204.8 \mathrm{~ms})$, the higher the image resolution. Figures 11(b)-11(d) illustrate three $2 \mathrm{D}$ images of the phantom constructed by the backprojection imaging method at the same acquisition rate $(100 \mathrm{kHz})$ but different acquisition times. As can be seen from Figures $11(\mathrm{~b}) \sim 11(\mathrm{~d})$, it is obvious that, with the increase of acquisition time, the image resolution is gradually improved, which is consistent with the calculated results.

Another phantom (Figure 12(a)), with the same size (outer diameter and length) as the one described above, was also used for the measurement. Several rods inside the phantom had different diameters of $3.5 \mathrm{~mm}, 3 \mathrm{~mm}, 2.5 \mathrm{~mm}$, $2.2 \mathrm{~mm}, 2 \mathrm{~mm}$, and $1.8 \mathrm{~mm}$. The reconstructed image (Figure 12(b)) shows that the rods inside the phantom with a diameter of $3.5 \mathrm{~mm}, 3 \mathrm{~mm}$, and $2.5 \mathrm{~mm}$ can be distinguished.

To demonstrate the highest image resolution of the ULF MRI system, a phantom consisting of twelve plastic rods with a diameter of $1.8 \mathrm{~mm}$ (Figure 12(c)) was used for the measurement. A typical phantom image was successfully reconstructed by the maximum likelihood expectation maximization (MLEM) algorithm based on the backprojection imaging method (Figure 12(d)) [33, 34]. The resolution of $1.8 \times 1.8 \mathrm{~mm}^{2}$ for the water phantom was obtained by performing 72 measurements. The total time to acquire this image is about 20 minutes. The imaging resolution and the acquisition time have been improved in comparison with the resolution of $2.5 \times 1.9 \mathrm{~mm}^{2}$ in an imaging time of about 26 minutes obtained previously by Inglis and co-workers [29]. Consequently, it is feasible to detect an object with a size of $1.8 \times 1.8 \mathrm{~mm}^{2}$ by the SQUID-based ULF MRI system. 


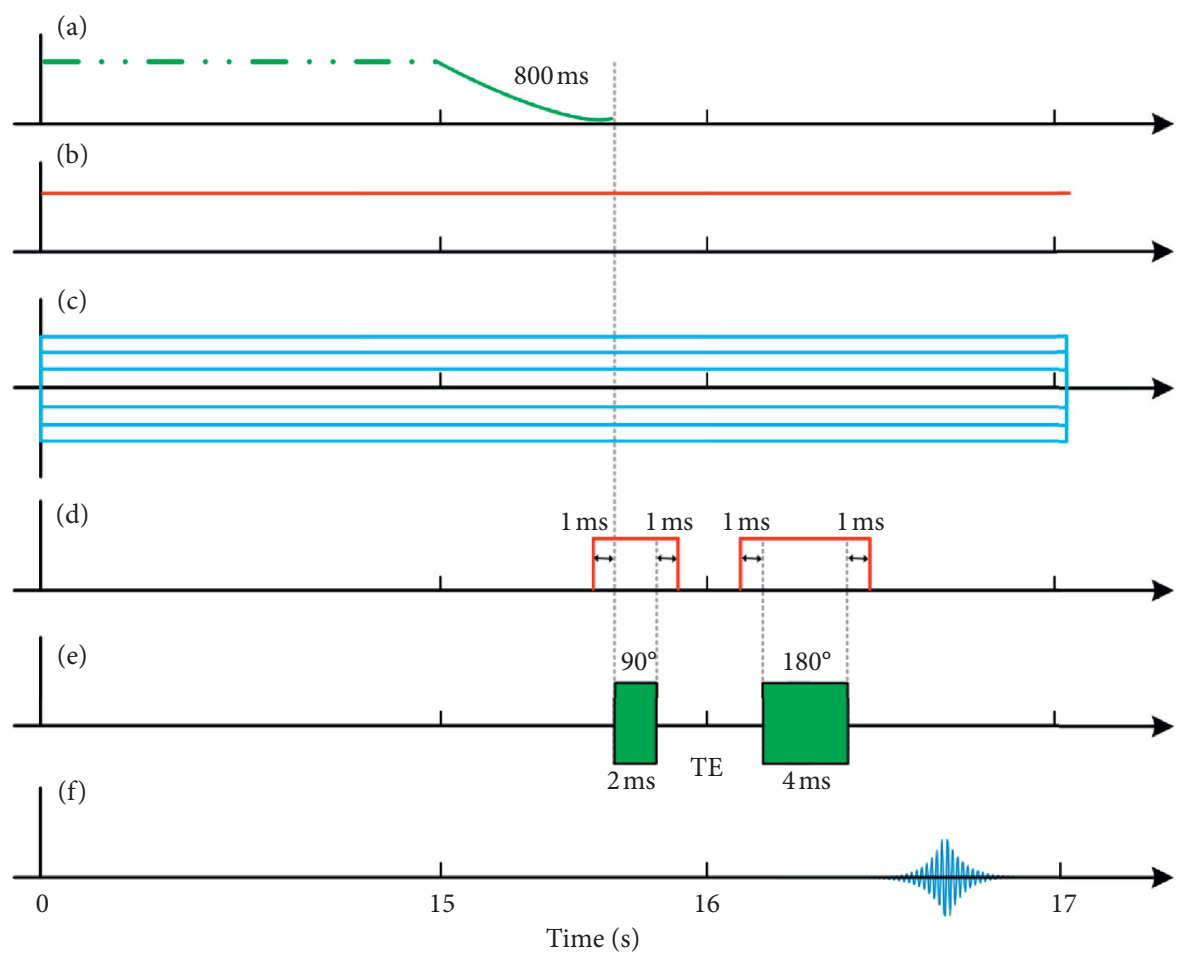

FIgURE 5: The pulse sequence used in the experiment for 2-D imaging. (a) $B_{p}$. (b) $B_{m}$. (c) $G_{y}$ and $G_{z}$. (d) SQUID CTRL. (e) $B_{1}$. (f) Signal.

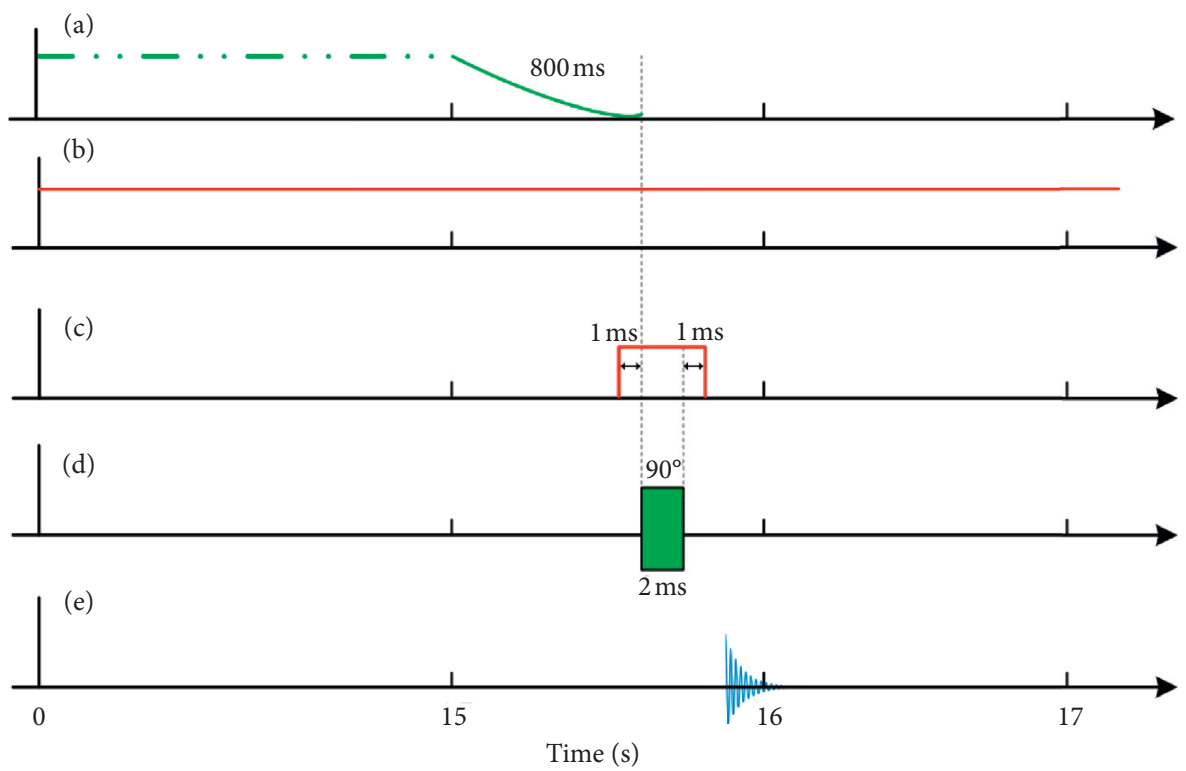

Figure 6: Pulse sequence for the detection of the FID signal. (a) $B_{p}$. (b) $B_{m}$. (c) SQUID CTRL. (d) $B_{1}$. (e) Signal.

\section{Discussion}

It is undeniable that MRI measurements at ULF play an increasing role in physics, chemistry, biology, and medicine for the lower cost of the ULF MRI system compared with conventional MRI. Although low-field MRI may never replace high-field scanners, there are certain applications in which its use may be exceedingly attractive. For example, the
ULF MRI approach may provide open MRI systems for airport security [11], emergency rooms, and field hospitals $[20,25]$, and it can also be combined with magnetoencephalography (MEG) for recording both anatomical and functional (biomagnetic) information about the brain $[23,24]$. Moreover, the unique advantage of ULF MRI is the higher $T_{1}$-contrast in low-field MRI which has been proposed for the discrimination of tissue [35-37]. This 


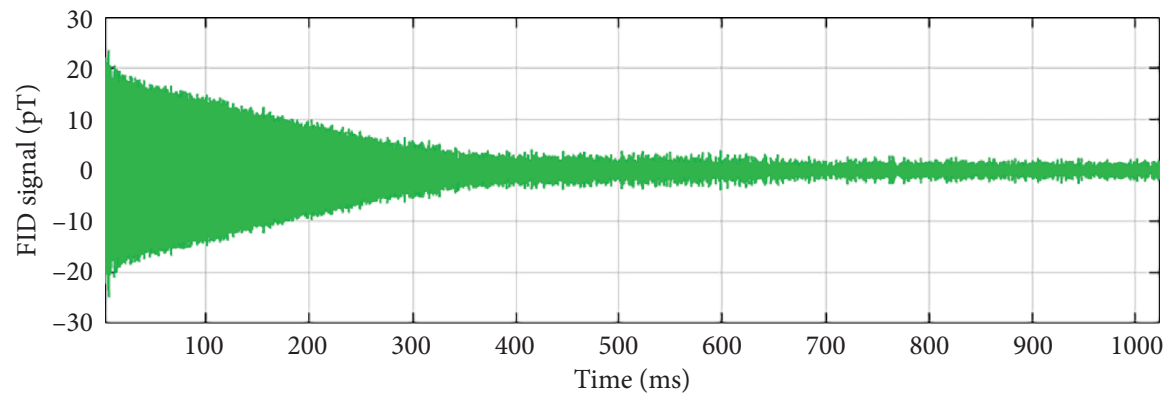

(a)

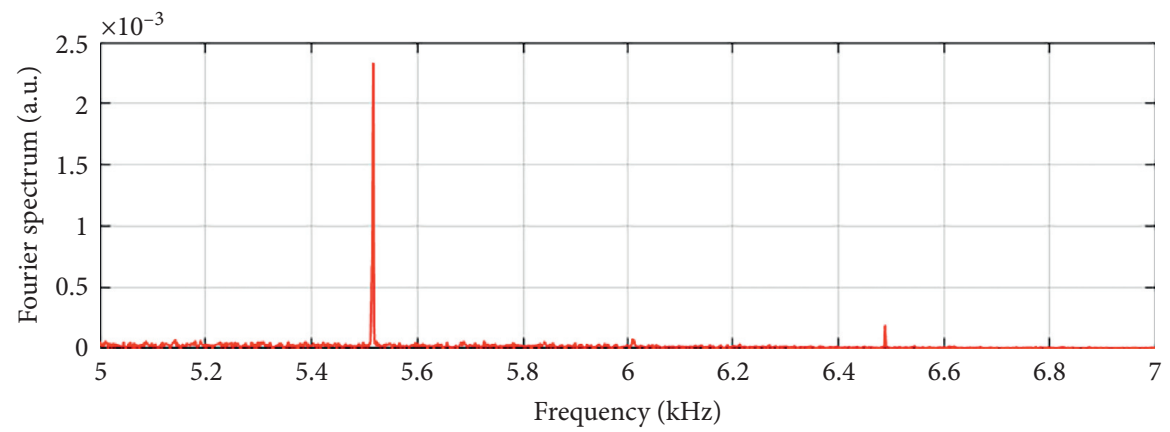

(b)

FIgURE 7: MRI measurement of a phantom containing pure water using the FID sequence. (a) FID signal without averaging. (b) The frequency spectrum of the FID signal.

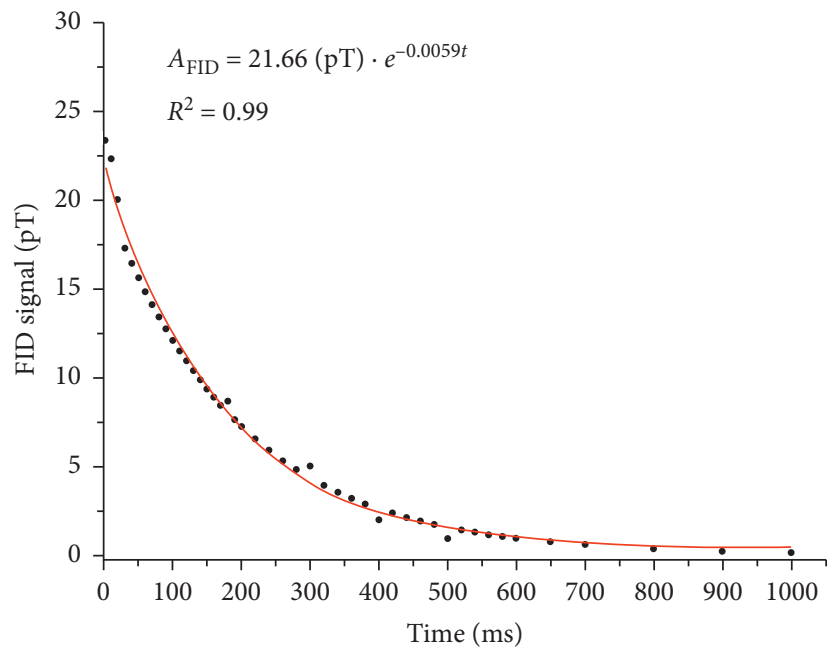

FIgURE 8: The fitting curve of the FID signal.

enhanced $T_{1}$-contrast is a prospective supplement to highfield MRI in pathology studies using the SQUID-based ULF MRI. It is also important in cases where there is no significant $T_{1}$-contrast between different tissue types in high magnetic fields [38].

The feasibility of SQUID-based ULF for MRI measurements based on the backprojection imaging method has been demonstrated in this paper. The backprojection method simplifies the imaging pulse sequence and consists of a series of projections of the sample in varying applied gradients at different angles. The gradient field for each projection does not need to be switched on or off in microseconds compared with Fourier imaging. However, it is necessary to maintain the low noise of the power supply since the gradient is constant during the measurement. Besides, the backprojection method can be extended to $3 \mathrm{D}$ imaging by simply applying a slice selection gradient pulse [30].

In this work, imaging experiments were conducted with three phantoms to characterize the performance of the ULF- 


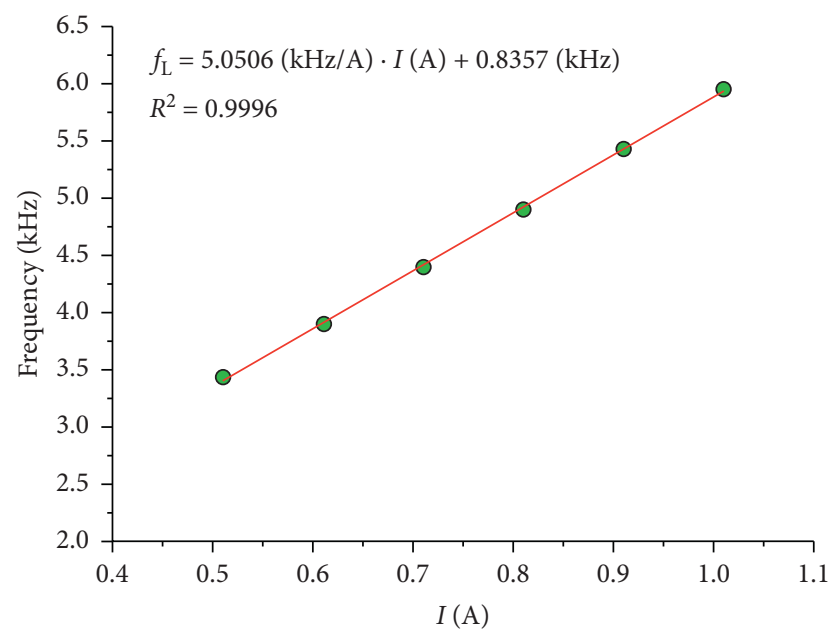

FIGURE 9: The measured Larmor frequency versus the current.

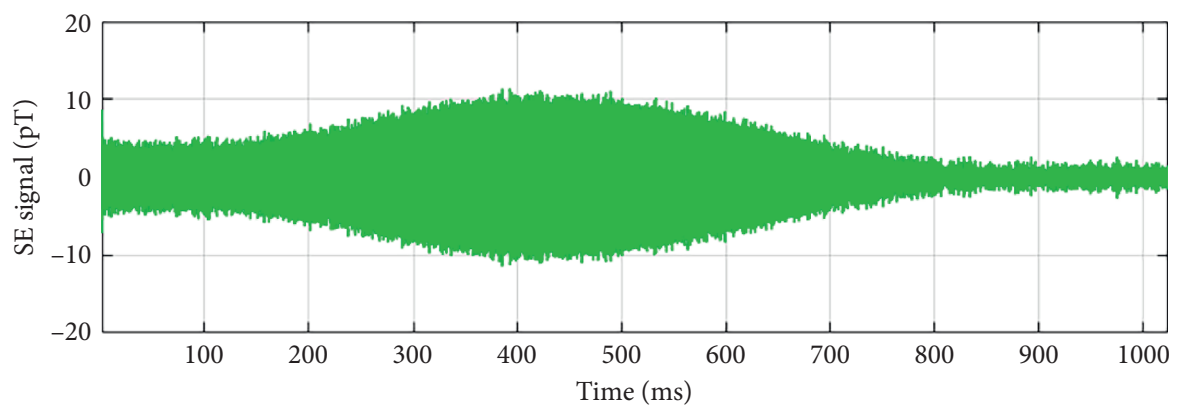

(a)

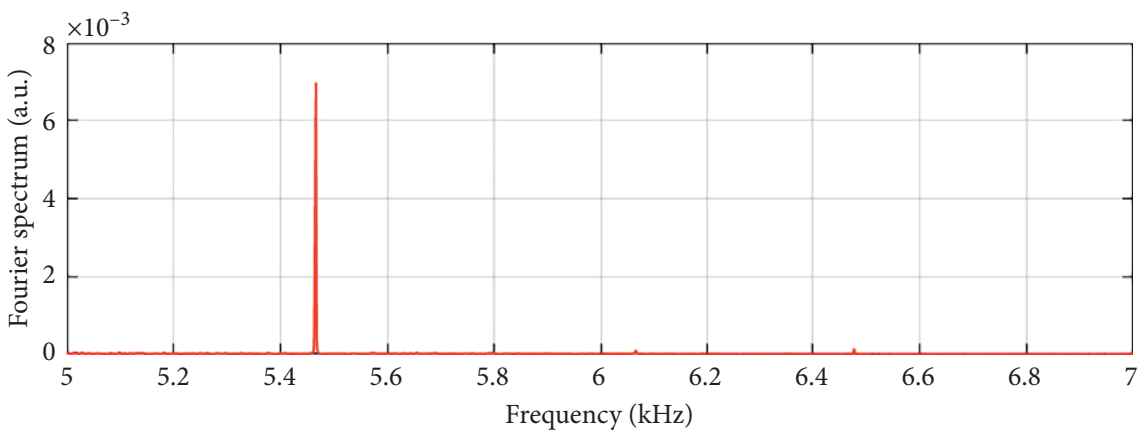

(b)

FIGURE 10: 1-D MRI measurements of a phantom filled with pure water using the SE sequence. (a) SE signal recorded without averaging. (b) The frequency spectrum corresponding to the SE signal.

MRI system by using multirods with different diameters inside the phantom. A $500 \mathrm{mT}$ prepolarization field was applied for the enhancement of MR signals. The results revealed that the system can image a water phantom with up to $1.8 \times 1.8 \mathrm{~mm}^{2}$ resolution which would make it possible to image the sample in the presence of metal. The constructed image (Figure 12) presented above is not well reproduced with the water phantom, which seems incomplete as if missing a part of the image. The reason for this phenomenon is that the water phantom was placed horizontally at the bottom of the SQUID. The upper part of the phantom is closer to the SQUID sensor, while the lower part is further from the SQUID, which leads to the SNR for the lower part being relatively smaller than for the upper part according to the Biot-Savart Law. Note that the vibration generated from the collision can also cause image artifacts during the transportation of samples.

For soft tissue measurement, the attenuation of the magnetization during the transport process is the main reason for limiting the detection of tissue with short 


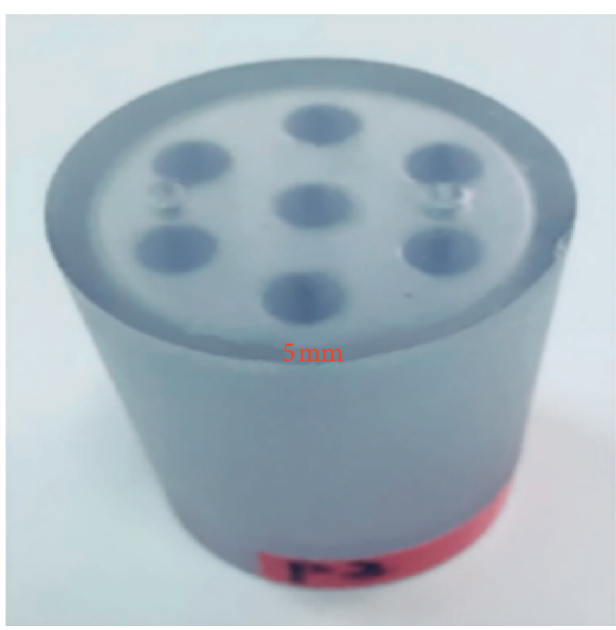

(a)

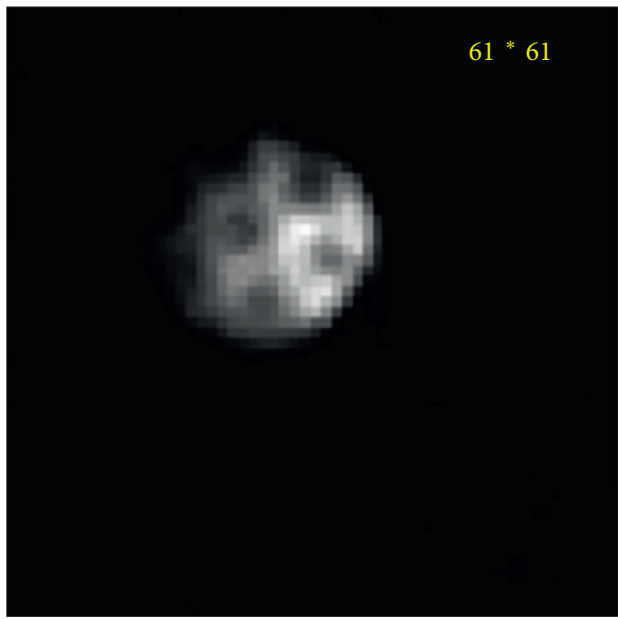

(c)

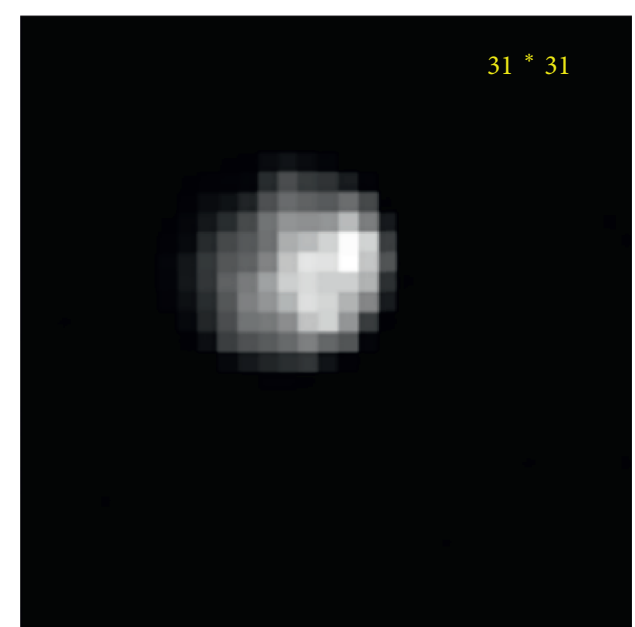

(b)

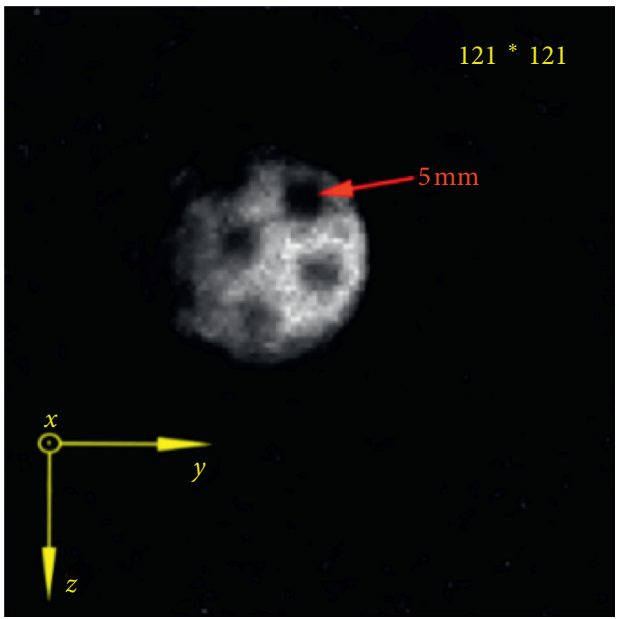

(d)

Figure 11: Images of the phantom with seven rods with a diameter of $5 \mathrm{~mm}$. (a) Photograph of a $35 \mathrm{~mm}$ diameter cylindrical plastic phantom. (b-d) 2-D image of the phantom reconstructed by the backprojection method at the acquisition time of $51.2 \mathrm{~ms}, 102.4 \mathrm{~ms}$, and $204.8 \mathrm{~ms}$, respectively. The images (b)-(d) were acquired with a prepolarization field $\left(B_{p}\right)$ of $500 \mathrm{mT}, B_{m}=128 \mu \mathrm{T}$, and the gradient was $\sim 180 \mu \mathrm{T} / \mathrm{m}$.

TABLe 1: The result of frequency resolution and image resolution calculated by varying the acquisition time.

\begin{tabular}{|c|c|c|}
\hline Acquisition time (ms) & Frequency resolution $(\mathrm{Hz})$ & Image resolution $(\mathrm{mm})$ \\
\hline 51.2 & 19.5 & 2.63 \\
\hline 102.4 & 9.8 & 1.28 \\
\hline 204.8 & 4.88 & 0.63 \\
\hline
\end{tabular}

relaxation time. A potential method could be used to increase the relaxation time by manipulating the molecule into a long-lived state [39]. Long-lived states allow spin magnetization to be stored for a considerably extended period of time, typically at least an order of magnitude longer than $T_{1}$ for the same molecule [40].

In future work, the prepolarization method may be improved by a polarizing coil around the measurement position. Therefore, the sample can be directly measured after prepolarization without the transport process, and the decay of the sample magnetization could be further reduced. This improved prepolarization technique provides the ULF MRI system a potential application for tissue imaging. The prepolarization field $B_{p}$ is also required to be increased to improve the quality of the reconstructed image of the sample. However, there are drawbacks in terms of induced eddy-currents from pulsing large magnetic fields inside a shielded room. Furthermore, programming a commercially available digital spectrometer could be attempted to implement 


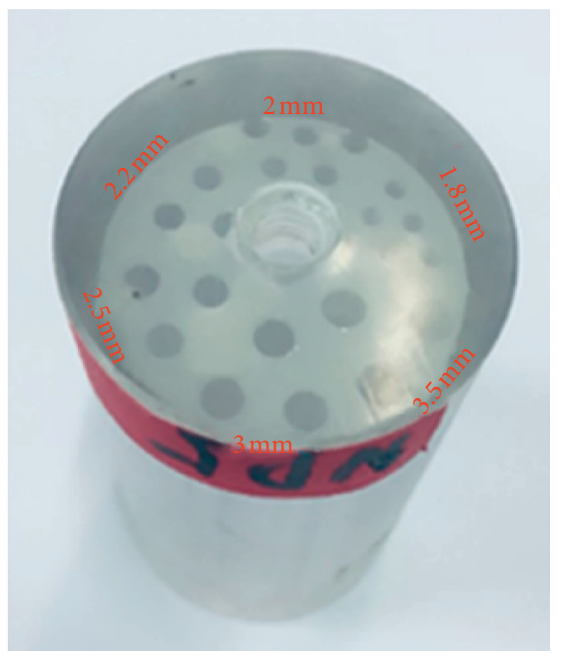

(a)

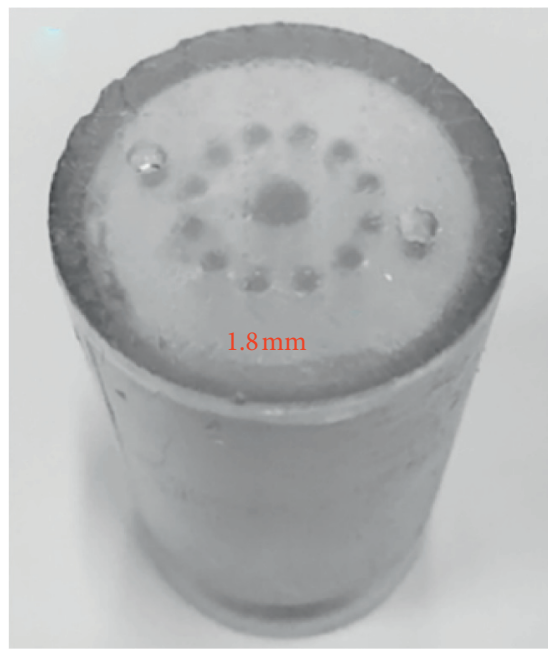

(c)

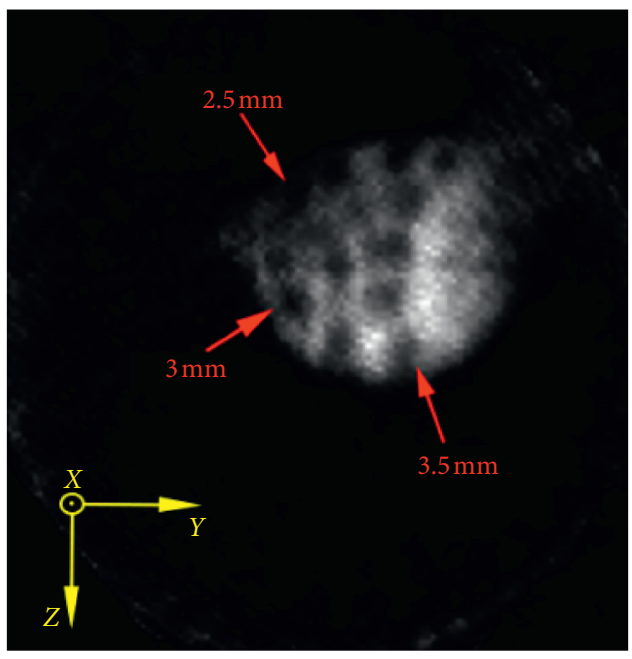

(b)

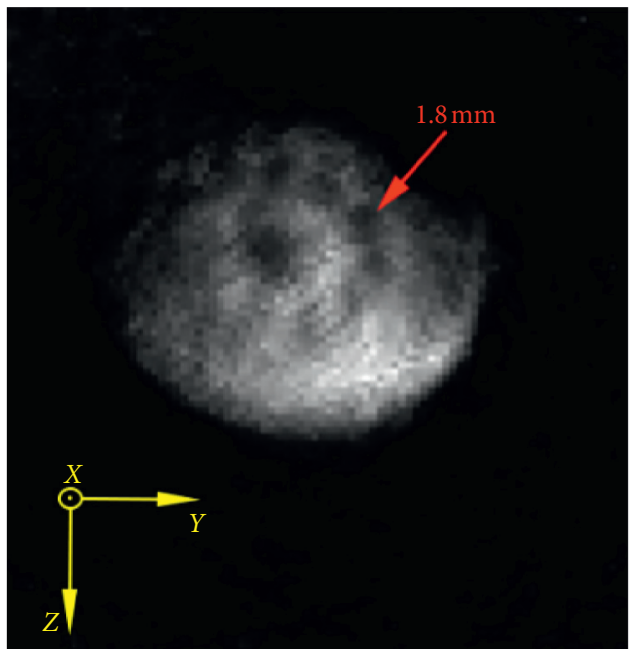

(d)

FIGURE 12: Images of a phantom containing several rods with different diameters. (a) Photograph of the phantom. (b) 2-D image of the phantom constructed by the backprojection method with rods of different diameters. Figure $12(\mathrm{~b})$ is acquired at the acquisition time of $256 \mathrm{~ms}$ with $B_{p}=500 \mathrm{mT}, B_{m}=128 \mu \mathrm{T}$, and the gradient was $\sim 160 \mu \mathrm{T} / \mathrm{m}$. (c) Photograph of a phantom with twelve $1.8 \mathrm{~mm}$ diameter rods. (d) 2-D image of the phantom constructed by the backprojection method with $1.8 \mathrm{~mm}$ diameter rods. Figure 12(d) was acquired at the acquisition time of $256 \mathrm{~ms}$ with $B_{p}=500 \mathrm{mT}, B_{m}=128 \mu \mathrm{T}$, and the gradient was $\sim 180 \mu \mathrm{T} / \mathrm{m}$.

more pulse sequences to increase the imaging resolution of the system.

\section{Conclusion}

We designed a SQUID-based MRI system and demonstrated the feasibility of MRI at the ultra-low field. The SNR obtained by applying the SE-based pulse sequence could reach up to 70 in a single measurement. The imaging resolution reached $1.8 \times 1.8 \mathrm{~mm}^{2}$ by using the maximum likelihood expectation maximization (MLEM) algorithm based on the backprojection method. Although ULF MRI has been significantly developed over the years, it still needs further improvement to enhance the imaging resolution within an acceptable time to complement high-field MRI.

\section{Data Availability}

The data used to support the findings of this study are available from the corresponding author (changy@sibet.ac.cn and xiaodong.yang@sibet.ac.cn) upon request.

\section{Conflicts of Interest}

The authors declare no conflicts of interest.

\section{Acknowledgments}

This work was supported by the National Natural Science Foundation of China (NSFC), Grant nos. 11675254 and 11505281, General Social Development Project of Jiangsu 
(BE2017670), and Chinese Academy of Sciences, Research and Development Project for Equipment, Grant no. YZ201445.

\section{References}

[1] C. Monninghoff, M. E. Ladd, and M. Forsting, "7-Tesla-MRI in neuroradiology possibilities and perspectives," Nervenheilkunde, vol. 32, no. 7, pp. 485-492, 2013.

[2] X. Hu and D. G. Norris, "Advances in high-field magnetic resonance imaging," Annual Review of Biomedical Engineering, vol. 6, no. 1, pp. 157-184, 2004.

[3] Y. Wang, F. Liu, E. Weber et al., "Acoustic analysis for a split MRI system using FE method," Concepts in Magnetic Resonance Part B: Magnetic Resonance Engineering, vol. 45, no. 2, pp. 85-96, 2015.

[4] B. Odintsov, J. L. Chun, J. A. Mulligan, and S. E. Berry, "14.1 T whole body MRI for detection of mesoangioblast stem cells in a murine model of Duchenne muscular dystrophy," Magnetic Resonance in Medicine, vol. 66, no. 6, pp. 1704-1714, 2011.

[5] C. Westbrook, C. K. Roth, and J. Talbot, MRI in Practice, Wiley-Blackwell, Hoboken, NJ, USA, 2011.

[6] R. R. Edelman, J. R. Hesselink, M. B. Zlatkin, and J. V. Crues, Clinical Magnetic Resonance Imaging, WB Saunders Co, Philadelphia, PA, USA, 3rd edition, 2006.

[7] B. Azzedine, M. B. Kahina, P. Dimitri, D. Alain, and M. Claude, "Whole-body diffusion-weighted MRI for staging lymphoma at 3.0T: comparative study with MRI imaging at 1.5T," Clinical Imaging, vol. 39, no. 1, pp. 104-109, 2014.

[8] K. Bahrami, F. Shi, I. Rekik, Y. Gao, and D. Shen, "7T-guided super-resolution of 3T MRI," Medical Physics, vol. 44, no. 5, pp. 1661-1677, 2017.

[9] J. S. Park, R. Vohra, T. Klussmann, N. E. Bengtsson, J. S. Chamberlain, and D. Lee, "Non-invasive tracking of disease progression in young dystrophic muscles using multiparametric MRI at 14T," PLoS One, vol. 13, no. 10, Article ID e0206323, 2018.

[10] M. Mößle, S. I. Han, W. R. Myers et al., "SQUID-detected microtesla MRI in the presence of metal," Journal of Magnetic Resonance, vol. 179, no. 1, pp. 146-151, 2006.

[11] A. N. Matlashov, L. J. Schultz, M. A. Espy et al., "SQUIDs vs. induction coils for ultra-low field nuclear magnetic resonance: experimental and simulation comparison," IEEE Transactions on Applied Superconductivity, vol. 21, no. 3, pp. 465-468, 2011.

[12] P. L. Volegov, A. N. Matlachov, and R. H. Kraus, "Ultra-low field NMR measurements of liquids and gases with short relaxation times," Journal of Magnetic Resonance, vol. 183, no. 1, pp. 134-141, 2006.

[13] J. Clarke, M. Hatridge, and M. Mößle, "SQUID-detected magnetic resonance imaging in microtesla fields," Annual Review of Biomedical Engineering, vol. 9, no. 1, pp. 389-413, 2007.

[14] L. Darrasse and J. C. Ginefri, "Perspectives with cryogenic RF probes in biomedical MRI," Biochimie, vol. 85, no. 9, pp. 915-937, 2003.

[15] H. C. Seton, J. M. S. Hutchison, and D. M. Bussell, "Liquid helium cryostat for SQUID-based MRI receivers," Cryogenics, vol. 45, no. 5, pp. 348-355, 2005.

[16] R. L. Fagaly, "Superconducting quantum interference device instruments and applications," Review of Scientific Instruments, vol. 77, no. 10, Article ID 101101, 2006.

[17] J. Dabek, P. T. Vesanen, K. C. J. Zevenhoven, J. O. Nieminen, R. Sepponen, and R. J. Ilmoniemi, "SQUID-sensor-based ultra-low-field MRI calibration with phantom images: towards quantitative imaging," Journal of Magnetic Resonance, vol. 224, pp. 22-31, 2012.

[18] M. Mossle, W. R. Myers, S. K. Lee et al., "SQUID-detected in vivo MRI at microtesla magnetic fields," IEEE Transactions on Applied Superconductivity, vol. 15, no. 2, pp. 757-760, 2005.

[19] J. Clarke and A. I. Braginski, The SQUID Handbook, Vol. 1, Wiley-VCH, Weinheim, Germany, 2004.

[20] M. Espy, M. Flynn, J. Gomez et al., "Ultra-low-field MRI for the detection of liquid explosives," Superconductor Science Technology, vol. 23, no. 3, p. 24, 2010.

[21] V. S. Zotev, A. N. Matlashov, I. M. Savukov et al., "SQUIDbased microtesla MRI for in vivo relaxometry of the human brain," IEEE Transactions on Applied Superconductivity, vol. 19, no. 3, pp. 823-826, 2009.

[22] R. McDermott, N. Kelso, S. K. Lee et al., "SQUID-detected magnetic resonance imaging in microtesla magnetic fields," Journal of Low Temperature Physics, vol. 135, no. 5-6, pp. 793-821, 2004.

[23] A. J. Mäkinen, K. C. J. Zevenhoven, and R. J. Ilmoniemi, "Automatic spatial calibration of ultra-low-field MRI for high-accuracy hybrid MEG-MRI," IEEE Transactions on Medical Imaging, vol. 38, no. 6, pp. 1317-1327, 2019.

[24] A. N. Matlashov, E. Burmistrov, P. E. Magnelind et al., "SQUID-based systems for co-registration of ultra-low field nuclear magnetic resonance images and magnetoencephalography," Physica C: Superconductivity and Its Applications, vol. 482, pp. 19-26, 2012.

[25] M. A. Espy, P. E. Magnelind, A. N. Matlashov et al., "Progress toward a deployable SQUID-based ultra-low field MRI system for anatomical imaging," IEEE Transactions on Applied Superconductivity, vol. 25, no. 3, pp. 1-5, 2015.

[26] M. Espy, A. Matlashov, and P. Volegov, "SQUID-detected ultra-low field MRI," Journal of Magnetic Resonance, vol. 229, pp. 127-141, 2013.

[27] X. Huang, H. Dong, Y. Qiu et al., "Adaptive suppression of power line interference in ultra-low field magnetic resonance imaging in an unshielded environment," Journal of Magnetic Resonance, vol. 286, pp. 52-59, 2018.

[28] M. W. Vogel, R. P. Guridi, J. Su, V. Vegh, and D. C. Reutens, "3D-spatial encoding with permanent magnets for ultra-low field magnetic resonance imaging," Scientific Reports, vol. 9, no. 1, Article ID 1522, 2019.

[29] B. Inglis, K. Buckenmaier, P. Sangiorgio, A. F. Pedersen, M. A. Nichols, and J. Clarke, "MRI of the human brain at 130 microtesla," Proceedings of the National Academy of Sciences, vol. 110, no. 48, pp. 19194-19201, 2013.

[30] R. McDermott, S. Lee, B. T. Haken, A. H. Trabesinger, A. Pines, and J. Clarke, "Microtesla MRI with a superconducting quantum interference device," Proceedings of the National Academy of Sciences, vol. 101, no. 21, pp. 7857-7861, 2004.

[31] H. Soltner and P. Blümler, "Dipolar Halbach magnet stacks made from identically shaped permanent magnets for magnetic resonance," Concepts in Magnetic Resonance Part A, vol. 36A, no. 4, pp. 211-222, 2010.

[32] W. R. Myers, Potential Applications of Microtesla Magnetic Resonance Imaging Detected Using a Superconducting Quantum Interference Device, pp. 20-26, University of California, Berkeley, CA, USA, 2006.

[33] T. Hu, Y. Wang, G. Du et al., "Improving the efficiency of small-angle $\mathrm{X}$-ray scattering computed tomography using the OSEM algorithm," Applied Optics, vol. 56, no. 30, pp. 8326-8334, 2017. 
[34] H. M. Hudson and R. S. Larkin, "Accelerated image reconstruction using ordered subsets of projection data," IEEE Transactions on Medical Imaging, vol. 13, no. 4, pp. 601-609, 1994.

[35] H. Yasui, S. Matsumoto, N. Devasahayam et al., "Low-field magnetic resonance imaging to visualize chronic and cycling hypoxia in tumor-bearing mice," Cancer Research, vol. 70, no. 16 , pp. 6427-6436, 2010.

[36] S. Busch, M. Hatridge, M. Mößle et al., "Measurements of $T_{1^{-}}$ relaxation in ex vivo prostate tissue at $132 \mu \mathrm{T}$," Magnetic Resonance in Medicine, vol. 67, no. 4, pp. 1138-1145, 2012.

[37] S. J. Lee, J. H. Shim, K. Kim et al., " $T_{1}$ relaxation measurement of ex-vivo breast cancer tissues at ultralow magnetic fields," Biomed Research International, vol. 2015, Article ID 385428, 9 pages, 2015.

[38] H. W. Fischer, P. A. Rinck, Y. Van Haverbeke, and R. N. Muller, "Nuclear relaxation of human brain gray and white matter: analysis of field dependence and implications for MRI," Magnetic Resonance in Medicine, vol. 16, no. 2, pp. 317-334, 1990

[39] Z. Zhou, K. Claytor, W. S. Warren, and T. Theis, "Accessing long lived $1 \mathrm{H}$ states via $2 \mathrm{H}$ couplings," Journal of Magnetic Resonance, vol. 263, pp. 108-115, 2016.

[40] M. H. Levitt, "Singlet nuclear magnetic resonance," Annual Review of Physical Chemistry, vol. 63, no. 1, pp. 89-105, 2012. 